Rabaska

Revue d'ethnologie de l'Amérique française

\title{
Benoît Lacroix (1915-2016)
}

\section{Jean Simard}

Volume 14, 2016

URI : https://id.erudit.org/iderudit/1037461ar

DOI : https://doi.org/10.7202/1037461ar

Aller au sommaire du numéro

Éditeur(s)

Société québécoise d'ethnologie

ISSN

1703-7433 (imprimé)

1916-7350 (numérique)

Découvrir la revue

Citer ce document

Simard, J. (2016). Benoît Lacroix (1915-2016). Rabaska, 14, 202-207.

https://doi.org/10.7202/1037461ar

Ce document est protégé par la loi sur le droit d'auteur. L'utilisation des services d'Érudit (y compris la reproduction) est assujettie à sa politique d'utilisation que vous pouvez consulter en ligne.

https://apropos.erudit.org/fr/usagers/politique-dutilisation/
Cet article est diffusé et préservé par Érudit.

Érudit est un consortium interuniversitaire sans but lucratif composé de l'Université de Montréal, l'Université Laval et l'Université du Québec à Montréal. Il a pour mission la promotion et la valorisation de la recherche. https://www.erudit.org/fr/ 


\section{Benoît Lacroix (1915-2016)}

Le 2 mars 2016 s'éteignait le père Benoît Lacroix, qui avait fêté ses 100 ans le 8 septembre précédent. En guise de notice nécrologique, Rabaska reproduit, avec l'aimable autorisation de Fernand Harvey, secrétaire de la Société des Dix, un article que je publiais dans les Cahiers des Dix en $1996^{1}$ sur la carrière de celui qui $m$ 'avait précédé au septième fauteuil de la vénérable Société fondée en 1935. Rabaska n'a pas attendu le décès de Benoît Lacroix pour accueillir des écrits sur son oeuvre : en 2007, son ancien confrère en religion, Pietro Boglioni, signait en nos pages un "Portrait » de Benoît Lacroix, tandis qu'en 2012 Giselle Huot publiait à son

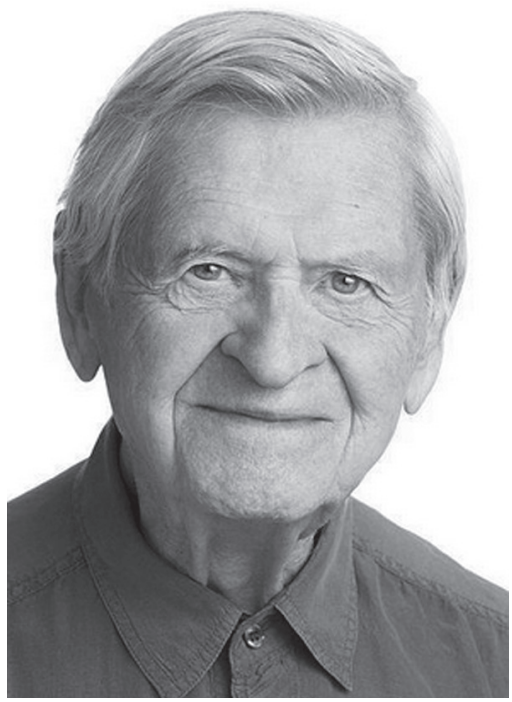

Martine Doyon, photographe, 11 août 2014 Source : agora.qc.ca/dossiers/Benoit Lacroix tour " La correspondance Luc Lacourcière - Benoît Lacroix (1950-1987) ». Benoît Lacroix lui-même nous avait donné en 2010 un point de vue sur le premier livre de Luc Lacourcière, Essais sur Émile Nelligan et sur la chanson populaire, intitulé "Luc Lacourcière, un homme partagé ».

La mort faisait-elle peur au père Lacroix? En tout cas pas les cimetières. Le 17 mars 2009, quelques mois après la sortie de mon livre Cimetières. Patrimoine pour les vivants (GID, 2008), il m'écrivait : " Depuis si longtemps quej'aime les cimetières; cette fois je suis admirablement bien servi. Victoire visuelle des patrimoines [...]. Finalement une certaine nostalgie traverse ces pages face à ces caveaux et colombariums, ces urnes si loin de la mer

1. Jean Simard, « Le septième fauteuil. Pierre-Georges Roy, Antoine Roy, Robert-Lionel Séguin, Benoît Lacroix », Cahiers des Dix, Sainte-Foy (Québec), Les Éditions La Liberté, 1996, p. $135-153$. 
et du fleuve [...]. Avant de terminer ma lettre, je relis Thérèse Labbé sur les femmes pleureuses, et je suis ému!»

Celui qui a occupé de 1982 à 1990 le fauteuil laissé libre par Robert-Lionel Séguin à la Société des Dix a fêté ses 80 ans le 8 septembre 1995. À cette occasion, 173 parents et amis lui ont offert, comme il semblait convenir à un religieux, un bouquet tout spirituel intitulé Dits et gestes de Benoît Lacroix. Prophète de l'amour et de l'esprit ${ }^{2}$, somme de témoignages adressés par ceux et celles qui ont côtoyé à un moment ou à un autre de leur vie, à un titre ou à un autre, cet homme qui a semé sur son chemin amour, foi et science. En ce dimanche du 24 septembre 1995, jour de lancement des Dits et gestes - dont le jubilaire semblait ignorer jusque-là l'existence -, le père Lacroix s'est plu à rappeler ses origines paysannes.

Le 8 septembre 1915, naissait à Saint-Michel-de-Bellechasse, de Caïus Lacroix et de Rose-Anna Blais, Joseph-Joachim-François-Xavier, dit plus tard Benoît. En 1927, Joachim quitte le Troisième rang ouest de Saint-Michel pour le collège de Sainte-Anne-de-la-Pocatière où il restera neuf ans. En 1936, il choisit de devenir dominicain et reçoit le nom de frère Benoît. On lui assigne pour patron le pape Benoît XI, dominicain lui-même, qui a régné à Rome de 1303 à 1304 et mourut probablement empoisonné. Joachim lui aurait bien préféré le grand saint Benoît de Nursie, le fondateur de l'ordre, mais son père maître en décida autrement : Benoît XI est « bien assez bon pour toi, lui dit-il, puisqu'il n'est, lui, contrairement à saint Benoît de Nursie, que bienheureux ${ }^{3} »$. Le père Lacroix apprend donc très tôt que l'humour aide à passer les épreuves. En 1937, le novice passe au Studium Generale des dominicains à Ottawa où il découvre pour maître à penser le père Louis-Marie Régis, « un pédagogue extraordinaire dont je n'ai jamais trouvé ailleurs l'équivalent [...]. Il était devenu sans doute mon père, un père idéal, un père instruit et diplômé $!^{4} »$. Études philosophiques et théologiques faites, il est ordonné prêtre le 5 juillet 1941. D’Ottawa, il passe plus au sud, à Toronto, au Pontifical Institute of Mediaeval Studies où il séjourne de 1941 à 1946. C'est là qu'il fait la connaissance de ses autres maîtres : «Mes modèles de compétence demeuraient le père Régis, Gilson, un peu moins Maritain et, quelques années plus tard, Henri-Irénée Marrou avec qui j’ai beaucoup travaillé ${ }^{5} \gg$. Il y prépare une licence (1944) puis un doctorat ès Sciences médiévales (1951) avec une thèse intitulée «Les débuts de l'historiographie médiévale. Ses origines. Son

2. Giselle Huot (dir.), Dits et gestes de Benoît Lacroix. Prophète de l'amour et de l'esprit, SaintHippolyte et Montréal, Éditions du Noroît et Fondation Albert-le-Grand, 1995, 735 p.

3. Ibid., p. 118.

4. Ibid.

5. Ibid., p. 153. 
esprit. Ses méthodes », le premier doctorat décerné par cet institut fondé en 1929. Depuis 1945, Benoît Lacroix était chargé de cours d'historiographie, de méthodologie et de paléographie à l'Institut d'études médiévales de l'Université de Montréal, fondé en 1942. Lorsqu'il obtient son doctorat, l'Université le nomme professeur agrégé. Il a 36 ans.

À partir de ce moment, le père Lacroix s'adonne à de multiples tâches que lui commande sa carrière. L'enseignement l'occupe bien entendu à plein temps, à l'Institut d'études médiévales d'abord, dont il dirige les destinées de 1963 à 1969, mais aussi à l'Université Laval (1955), à l'Université nationale de Kyoto au Japon (1961), à l'Université nationale du Rwanda (1965) qu'avait fondée son confrère dominicain Georges-Henri Lévesque, à l'Université de Caen (1973-1976). Il fait des séjours d'étude à l'étranger. En 1957, il est au Centre d'études supérieures de civilisation médiévale de l'Université de Poitiers pour y étudier l'art roman; en 1959, il obtient une bourse de la Guggenheim Foundation de New-York pour étudier la philosophie de l'histoire. C'est là qu'il écrit sur Orose et ses idées ${ }^{6}$. Il écrit donc sur ce qu'il enseigne, mais on le sollicite de toutes parts. Les éditeurs de ses Dits et gestes ont bien montré la variété de ses productions dans le seul domaine de l'écriture : histoire de la culture populaire (59 textes), théologie (53), critique littéraire, éditoriaux et préfaces (37), littérature, contes, narrations, poésie (32), histoire (20), édition et bibliographie (10). En 1967, il fait paraitre dans la collection " Classiques canadiens ", chez Fides, une synthèse sur Lionel Groulx, qu'il considère comme son dernier grand maître. Quelques années auparavant, le père Lacroix avait soumis au chanoine lui-même l'idée de publier ses œuvres complètes. En 1967, il revient à la charge. « Je m'intéresse au projet parce qu'il est de vous », lui répond Groulx. « Je ne puis me défendre d'un certain désintéressement puisque je n'en verrai, selon toute probabilité, ni le commencement ni la fin ${ }^{7} \gg$. L'œuvre est l'une des plus monumentales qui soient au Québec et même au Canada, assure Juliette Lalonde-Rémillard, qui a consacré sa vie entière à la carrière puis à la fortune critique des écrits de son oncle historien, mais on s'y attaque. À ce jour, est paru le Journal, en deux volumes (1984) et deux des quinze tomes prévus de la Correspondance (1989 et 1993). Giselle Huot, Juliette Lalonde-Rémillard et Pierre Trépanier en ont été les éditeurs, Benoît Lacroix l'animateur et le préfacier. Le père Lacroix ne délaisse pas le Moyen Âge pour autant, en tout cas pas complètement.

Une idée le hante depuis 1950. Il entend un jour démontrer que le Canada français a la même dette envers le Moyen Âge que la France des Français :

6. Benoît Lacroix, Orose et ses idées, Paris, J. Vrin et Montréal, Institut d'études médiévales, $1965,235 \mathrm{p}$.

7. Juliette Lalonde-Rémillard, «Édition des CEuvres de Lionel Groulx. Hommage à un homme d'action », Dits et gestes, op. cit., p. 316. 
« Nous sommes, je le répète, des transplantés d'outre-Atlantique, des "survivants" de l'Europe. Notre histoire "américaine" commence chronologiquement parlant au Moyen Âge ( $\mathrm{XV}^{\mathrm{e}}$ siècle), et notre histoire "canadienne" débute exactement au moment où celle du Moyen Âge s'achève : au XVI ${ }^{\mathrm{e}}$ siècle. Même le $\mathrm{XVI}^{\mathrm{e}}$, dont nous dépendons plus directement, est plus médiéval qu'on ne le dit habituellement. M. Gilson l'a montré pour la philosophie et Focillon pour l'art [...]. Le Moyen Âge est fortement inscrit en tout Canadien français dont les ancêtres remontent au régime français. Le caractère robuste et tendre à la fois de nos paysans, leur force physique qui fait échec à une extrême sensibilité ; tout ce qui relève de leur vie sociale ; par exemple, la politesse un peu prétentieuse parfois et souvent rituelle de plusieurs de nos bonnes gens, résidu des vieilles habitudes de cour et de la chevalerie, ces qualités d'ordre et de mesure, ce besoin d'équilibre au sein même de l'ardeur, cet idéalisme foncier ; ce goût aussi pour les idées, pour la logique, pour la dialectique, qui se transpose dans les conversations les plus ordinaires, dans les discussions et dans les débats politiques, tout cela est médiéval d'abord ${ }^{8} »$. En 1950, il se contentait de l'affirmer comme une intuition. Plus tard il souhaiterait pouvoir le démontrer en mettant en train un vrai programme de recherche : « Une enquête dans tous les domaines de la pensée aboutirait aux mêmes conclusions [...]. L'histoire de nos croyances, celle de notre folklore, de nos habitudes, de nos coutumes et de notre langue, le prouverait ${ }^{9} »$.

Il pose un premier geste en 1967, à Saint-Michel-de-Bellechasse, en interviewant son père et sa sœur aînée Marie-Jeanne sur les coutumes funéraires anciennes dans Bellechasse. Dans les mois qui suivent, il multiplie les entrevues enregistrées auprès de personnalités issues de la biologie, de l'histoire, de la sociologie, de la psychologie, de l'ethnologie, de la muséologie. Il prépare la fondation du Centre d'études des religions populaires qui aura pour objet de retisser les liens entre le Moyen Âge et le Québec. Il met les gens au travail, surtout des étudiants de l'Institut d'études médiévales qui se préparent au doctorat. À Giselle Huot, qui est rendue à l'Université d'Aixen-Provence, il écrit : «J'ai nettement l'impression que votre connaissance du Moyen Âge et des méthodes que vous apprendrez là-bas sur l'étude des mentalités religieuses vous habilitent déjà à voir large et grand. Il y a le fait religieux du Moyen Âge européen et il y a le fait religieux du Moyen Âge d'ici. Celui-ci se termine à peine. Que faire $?^{10} »$. Sitôt collectées, les données sont transcrites dans une publication maison, les Cahiers d'études des religions populaires. Le Cahier XII de 1971 propose un questionnaire de

8. Benoît Lacroix, « Pourquoi aimer le moyen âge ? », ibid., p. 142.

9. Ibid.

10. Benoît Lacroix, « Journal des débats du Centre d'études des religions populaires (extraits de lettres) », Dits et gestes, p. 224. 
150 entrées sur « La religion de mon enfance » : Adoration nocturne, Anges, Chandeleur, Deuil, Enfer, Fin du monde, etc. Il ne suffisait pas de collecter, il fallait aussi étudier. Une série de colloques internationaux est donc mise sur pied par Benoît Lacroix. Il y en aura onze en douze ans, dans la plupart des villes du Québec ainsi qu'à Moncton, à Ottawa et à Sudbury, de 1970 à 1982. Celui de 1982 fut tenu à l'Université Laval. Le père Lacroix recrutait toujours des partenaires locaux. Il fit appel à Jean-Paul Montminy, à Fernand Dumont, à Pierre Savard et à moi pour la structuration du programme. Nous en avons publié ensuite les actes ${ }^{11}$, comme il avait été fait pour la majorité des colloques précédents. Nul ne pourra dire si ces discussions ont démontré noir sur blanc le lien de causalité historique entre le Moyen Âge et nous. Une chose est cependant certaine : cette grande croisade sur l'étude de la religion populaire aura suscité un nombre important de travaux et même des vocations scientifiques, des échanges fructueux, voire cordiaux, entre disciplines et collègues du Canada, d'Europe et des États-Unis.

En 1981, Benoît Lacroix est nommé professeur émérite de son université. C'est l'âge de la retraite puisqu'il a maintenant 66 ans; en tout cas certains ont pu le penser. Il est de plus en plus « tout à tous », comme au temps de la JÉC au collège de Sainte-Anne-de-la-Pocatière. Mais surtout il tombe dans l'écriture. Dans le champ de la théologie « populaire », il avait commis quatre écrits ; depuis 1980, c'est 56. Dans celui de la religion populaire, c'était 14 avant 1980 ; après ce sera 42. Il convient de préciser aussi que pendant sa carrière universitaire il écrit principalement sur le Moyen Âge et en histoire, alors qu'après ce sera en homme d'Église et sur l'étude des religions populaires ${ }^{12}$. Il devient membre des Dix en 1982 et n'y publiera qu'un seul article : «Lionel Groulx en $1930^{13} »$. Pendant les huit ans de son séjour dans la Société, Les Cahiers des Dix ne paraissent qu'une fois. La reprise se fait en 1989 quand les Éditions La Liberté rachètent le fonds et stabilisent la situation financière.

11. Benoît Lacroix et Jean Simard (dir.), Religion populaire, religion de clercs ?, Québec, Institut québécois de recherche sur la culture, 1984, 444 p.

12. Au moment de préparer la réédition de cette notice biographique, Fenand Harvey me rappelle que le père Lacroix a occupé les premières années de sa retraite (1980-1986) à s'investir au comité scientifique de l'Institut québécois de recherche sur la culture (IQRC), organisme créé en 1979 et dirigé alors par le sociologue Fernand Dumont. En 1980, il prend la direction d'un groupe - composé de Lucille Côté, Hélène Dionne, Michèle Trudel-Drouin, Danielle Nepveu et Louise Rondeau - chargé d'un vaste programme de recherche sur la religion populaire. Les publications suivantes de l'IQRC émanent de cette initiative : Benoît Lacroix et Jean Simard, Religion populaire, religion de clercs ? (1984) ; Benoît Lacroix et Madeleine Grammond, Religion populaire au Québec. Typographie des sources : Bibliographie sélective (1900-1980) (1985) ; Benoît Lacroix, La Religion de mon père (1986). Il participe également à d'autres initiatives de l'IQRC, notamment le concours Mémoire d'une époque et le projet d'anthropologie religieuse. Le père Benoît participe en outre à plusieurs émissions de radio et de télévision sur ces questions et il fait paraître en traduction espagnole : Tipologia en la religiosidad popular en Canada, La Antigua, Universidad Santa Maria la Antigua, Panama.

13. Benoît Lacroix, «Lionel Groulx en 1930 », Les Cahiers des Dix, vol. 44, 1989, p. 199-229. 
J'ai eu l'honneur de succéder au père Lacroix au fauteuil numéro 7 de la Société des Dix en 1991. Nous avions organisé ensemble le dernier colloque sur les religions populaires en 1982 ; l'année d'après, nous réfléchissions avec Michel Lessard et d'autres collègues à un musée des religions qui a vu le jour en 1986 à Nicolet ; c'est lui qui me présenta à la Société royale du Canada où je fus élu en 1995. Nous avons eu pendant toutes ces années des contacts fréquents desquels j'ai retiré chaque fois des leçons de vie. J'ai appris surtout du père Lacroix que l'intelligence et la sensibilité, la science et l'intuition, la théorie et le terrain devaient faire bon ménage pour que la « vraie vérité » jaillisse quelque part. Son exemple me guidera toujours.

JEAN SimARD Université Laval 